\title{
A SIMPLE PROOF OF A RESULT IN FINITE PLASTICITY*
}

\author{
BY \\ J. CASEY \\ University of Houston
}

\begin{abstract}
Summary. Within the framework of a purely mechanical rate-type theory of finitely deforming elastic-plastic materials, a simple proof is given of a normality condition which has been shown by Naghdi \& Trapp [6] to follow from a physically plausible work assumption. In addition, a variation of the proof is used to demonstrate convexity of yield surfaces for a special class of materials, a result which was also originally established by Naghdi \& Trapp [10].
\end{abstract}

1. Introduction. Work inequalities have played an important role in the development of the theory of plasticity. Indeed, a unified approach to infinitesimal plasticity was first made possible by Drucker's postulate [1] concerning the nonnegativity of work in a cycle of stress. With the use of this postulate, restrictions on constitutive equations could be derived in a systematic way. In particular, Drucker's postulate provided a rigorous means of establishing the convexity of the yield surface in stress space as well as the result that the plastic strain rate is directed along the normal to the yield surface in stress space. A detailed account of Drucker's postulate and its consequences, together with proofs, may be found in the survey article by Naghdi [2].

Another work postulate, also stated in the context of small deformations, but this time involving a cycle of strain was proposed by Il'iushin [3]. As noted in [3], Il'iushin's postulate is less restrictive than that of Drucker.

A general thermodynamical theory of finitely deforming rate-independent elastic-plastic materials was presented by Green \& Naghdi [4, 5]. The corresponding purely mechanical development is contained in the paper of Naghdi \& Trapp [6], which incorporates those parts of $[4,5]$, specialized to the isothermal case, that are independent of the ClausiusDuhem entropy production inequality. With the object of deriving more specific restrictions on the general constitutive equations of $[4,5]$ than was possible using thermodynamical arguments, a physically plausible work assumption was introduced in [6]. This assumption concerns the nonnegativity of external work done on a body in a cycle of homogeneous deformation, and leads to a work inequality involving the stress power. In the context of small deformations, when no distinction is made between different measures of stress, the latter inequality has the same form as that of Il'iushin.

${ }^{*}$ Received November 16, 1982. 
A number of important consequences follow from the work assumption of Naghdi \& Trapp. Foremost among these is the result that a certain second order tensor $\sigma_{K L}$, which depends on elastic-plastic response functions, is necessarily directed along the inward normal to the yield surface in strain space [6]. It should be emphasized that $\sigma_{K L}$ is not parallel to the plastic strain rate tensor, except in the case of special constitutive equations. The normality of $\sigma_{K L}$ in turn implies that the basic measure of strain-hardening under multiaxial loading is related to the inner product of the normal to the yield surface in strain space and the normal to the yield surface in stress space [7, 8, 9]. Further consequences of the work assumption were established in [10], where in particular it was shown that for a special class of elastic-plastic materials, both the yield surface in strain space and the yield surface in stress space are convex.

In connection with the development in [6], it is pertinent to add that while no explicit use was made of loading criteria, the main discussion was conducted in a strain space setting. The question therefore arose as to whether the stress space formulation utilized in $[4,5]$, and in particular the loading criteria, might not be advantageously replaced by a strain space formulation. This matter was investigated by Naghdi \& Trapp [11], who showed that in fact, the stress space formulation has certain defects which can be remedied by an alternative strain space formulation. A summary of $[6,10,11]$ may be found in [12].

While the main result of [6], i.e. the normality of $\sigma_{K L}$, is appealing in its simplicity, the method by which this result is derived is rather complicated and involves detailed estimates of the various contributions to the work inequality for a finite cycle of deformation. A more readily accessible proof is given in the present paper. As in [6], a family of special finite strain cycles is employed here also, but the cycles are simpler and the work done on the cycles is estimated in a more direct way. It is especially transparent from the present method of derivation as to why the result holds: the dominant part of the "elastic" contribution' to the work inequality is cancelled during the return portion of the cycle.

Necessary background material on the strain space formulation of plasticity is summarized in Sec. 2 and the proof is contained in Sec. 3. In Sec. 4, after first illustrating the consequences of the normality of $\sigma_{K L}$ for a restricted set of constitutive equations, a variation of the proof given in Sec. 3 is used to demonstrate convexity of yield surfaces in a special case.

2. Background material. Consider a deformable elastic-plastic continuum moving in a three-dimensional Euclidean space. Let the components of the Lagrangian strain, plastic strain and symmetric Piola-Kirchhoff stress tensors be denoted by $E_{K L}, E_{K L}^{p}$ and $S_{K L}$, respectively, and let $\kappa$ be the work-hardening parameter. Geometrically, $E_{K L}$ and $S_{K L}$ may be regarded as the coordinates of points in six-dimensional Euclidean strain space and stress space, respectively.

\footnotetext{
'I.e. the part which contains the function $h(t)$ in (3.6),
} 
Suppose that the stress is specified by a constitutive equation of the form $[4,5]$

$$
S_{K L}=\hat{S}_{K L}\left(E_{M N}, E_{M N}, \kappa\right) .
$$

For brevity, let

$$
L_{K L M N}=\frac{\partial \hat{S}_{K L}}{\partial E_{M N}}=L_{L K M N}=L_{K L N M}
$$

In addition, assume the existence of a smooth scalar-valued yield function $g\left(E_{M N}, E_{M N}, \kappa\right)$ such that for fixed values of $E P_{N}$ and $\kappa$, the equation $g=0$ represents a closed orientable yield surface of dimension five enclosing the elastic region $(g<0)$ in strain space [11].

For any given motion, one may associate with each particle $X$ of the elastic-plastic body a smooth oriented curve $C_{e}$ which lies in strain space and is parametrized by time; $C_{e}$ will be referred to as a strain trajectory. The six-dimensional tangent vector at a point of $C_{e}$ is $\dot{E}_{K L}$, where a superposed dot signifies material time differentiation. The inner product of the tangent to $C_{e}$ and the outward unit normal to the yield surface is given in the usual form by

$$
\hat{g}=\frac{\partial g}{\partial E_{K L}} \dot{E}_{K L}
$$

evaluated at $g=0$.

As in [7], a strain space formulation is adopted as primary. The constitutive equations for the rate of plastic strain and the rate of work-hardening are then given by [11]

$$
\dot{E}_{K L}^{p}=\left\{\begin{array}{lll}
0 & \text { if } g<0, & \text { (a) } \\
0 & \text { if } g=0 \text { and } \hat{g}<0, & \text { (b) } \\
0 & \text { if } g=0 \text { and } \hat{g}=0, & \text { (c) } \\
\lambda \rho_{K L} \hat{g} & \text { if } g=0 \text { and } \hat{g}>0, & \text { (d) }
\end{array}\right.
$$

and

$$
\dot{\kappa}=e_{K L} \dot{E}_{K L},
$$

where $\lambda$ is a scalar-valued function of $\left(E_{M N}, E_{M_{N}}, \kappa\right)$, and $\rho_{K L}$ and $\mathcal{C}_{K L}$ are tensor-valued functions of the same variables. The conditions involving $g$ and $\hat{g}$ in (2.4) are the loading criteria of the strain space formulation and in the order listed correspond to: (a) an elastic state; (b) unloading from an elastic-plastic state; (c) neutral loading from an elastic-plastic state; and (d) loading from an elastic-plastic state. The following geometrical interpretation may be given [7]: In an elastic state the strain trajectory $C_{e}$ lies in the elastic region. During unloading, $C_{e}$ intersects the yield surface and points into the elastic region. During neutral loading, $C_{e}$ continues to lie on the yield surface. In these three cases, the yield surface remains stationary. During loading, $C_{e}$ intersects the yield surface and is pointing outwards. It is stipulated by the "consistency condition" that in this case the yield surface is pushed outwards by $C_{e}$, so that during loading $g$ remains equal to zero, and hence $\dot{g}$ is 
zero also. It then follows that ${ }^{2}$

$$
1+\lambda \rho_{K L}\left\{\frac{\partial g}{\partial E_{k L}^{p}}+\frac{\partial g}{\partial \kappa} \bigodot_{K L}\right\}=0, \quad \lambda>0
$$

and that not all components of $\rho_{K L}$ are zero. An elastic-plastic material is defined by prescribing the constitutive functions $\hat{S}_{K L}, \rho_{K L}, \mathcal{C}_{K L}$ and $g$. The Lagrange multiplier $\lambda$ may be calculated from (2.6).

Along a strain trajectory $C_{e}$ it follows from (2.1), (2.2), (2.4 a, b, c, d) and (2.5) that in an elastic state, and during unloading and neutral loading

$$
\dot{S}_{K L}=L_{K L M N} \dot{E}_{M N},
$$

whereas during loading

$$
\begin{aligned}
\dot{S}_{K L} & =L_{K L M N} \dot{E}_{M N}+\lambda \hat{g} \sigma_{K L} \\
& =\left\{L_{K L M N}+\lambda \sigma_{K L} \frac{\partial g}{\partial E_{M N}}\right\} \dot{E}_{M N},
\end{aligned}
$$

where the abbreviation

$$
\sigma_{K L}=\left\{\frac{\partial \hat{S}_{K L}}{\partial E P_{M N}}+\frac{\partial \hat{S}_{K L}}{\partial \kappa} \mathcal{C}_{M N}\right\} \rho_{M N}
$$

has been introduced.

With particular reference to elastic-plastic materials, the work assumption of Naghdi \& Trapp [6] may be stated as: The external work done on an elastic-plastic body by surface tractions and body forces in any sufficiently smooth homogenous ${ }^{3}$ cycle of deformation is nonnegative.

Suppose that at any time $t_{1}$, a homogeneous elastic-plastic body has values of strain, plastic strain and work-hardening parameter which are independent of $X$. Let the value of the strain be denoted by $E_{K L}^{0}$. Suppose in addition that the body is subjected to a sufficiently smooth homogeneous cycle of deformation which ends at time $t_{2}$. For this cycle, the strain trajectory $C_{e}$ is a closed curve beginning at $E_{K L}^{0}$ at time $t_{1}$ and returning to $E_{K L}^{0}$ at time $t_{2}$. It was shown in [6] that for any such cycle, the work assumption of Naghdi \& Trapp implies that ${ }^{4}$

$$
\int_{t_{1}}^{t_{2}} S_{K L} \dot{E}_{K L} d t \geqslant 0
$$

\footnotetext{
${ }^{2}$ For further details, see [9].

${ }^{3}$ Recall that in a homogeneous deformation, the deformation gradient, and hence also the Lagrangian strain, is independent of $X$.

${ }^{4}$ An inequality of the form (2.9), without the equality sign, is the starting point of Il'iushin's development [3] which, however, is confined to small deformations of a special class of materials. It should also be noted that Il'iushin considers his inequality to be synonymous with plastic behavior; in contrast, Naghdi \& Trapp [6] regard their work assumption not as a definition of plastic behavior, but as an additional restriction on the response of elastic-plastic materials.
} 
Since $\dot{E}_{K L}^{0}=0$ and $E_{K L}\left(t_{1}\right)=E_{K L}\left(t_{2}\right)=E_{K L}^{0}$, an integration by parts leads to

$$
\int_{t_{1}}^{t_{2}} \dot{S}_{K L}\left(E_{K L}-E_{K L}^{0}\right) d t \leqslant 0 .
$$

By applying (2.10) to an infinite sequence of nested strain cycles, Naghdi \& Trapp [6] deduced that the tensor $\sigma_{K L}$ is directed along the inward normal to the yield surface in strain space: ${ }^{5}$

$$
\sigma_{K L}=-\gamma^{*} \frac{\partial g}{\partial E_{K L}}, \quad \gamma^{*} \geqslant 0,
$$

where $\gamma^{*}$ is an undetermined scalar-valued function of $\left(E_{M N}, E_{M N}, \kappa\right)$. In the next section, (2.11) will be proved by a simple argument.

For later reference, we note that the substitution of $(2.11)$ into $(2.7 \mathrm{~b})$, leads to the result that during loading

$$
\dot{S}_{K L}-L_{K L M N} \dot{E}_{M N}=-\lambda \gamma^{*} \hat{g} \frac{\partial g}{\partial E_{K L}} .
$$

Furthermore, upon contraction of both sides of (2.12) with $\dot{E}_{K L}$, it follows that

$$
\dot{S}_{K L} \dot{E}_{K L} \leqslant L_{K L M N} \dot{E}_{K L} \dot{E}_{M N} \text {. }
$$

3. Proof of the normality of $\sigma_{K L}$. Choose $t_{1}=0$ and let $E_{K L}^{0}$ be any point on the yield surface. Also, let $m_{K L}$ be a unit vector in strain space, such that on the yield surface

$$
\frac{\partial g}{\partial E_{K L}} m_{K L}>0 \quad\left(m_{K L} m_{K L}=1\right)
$$

Consider the following special homogeneous cycle:

$$
E_{K L}(t)= \begin{cases}E_{K L}^{0}+t m_{K L}, & 0 \leqslant t \leqslant \varepsilon, \\ E_{K L}^{0}+(2 \varepsilon-t) m_{K L}, & \varepsilon \leqslant t \leqslant 2 \varepsilon,\end{cases}
$$

where $\varepsilon$ is any positive real number which is sufficiently small that $E_{K L}^{0}$ still lies in the elastic region at time $\varepsilon$. Clearly,

$$
\dot{E}_{K L}(t)= \begin{cases}m_{K L}, & 0 \leqslant t<\varepsilon \\ -m_{K L}, & \varepsilon<t \leqslant 2 \varepsilon\end{cases}
$$

Also, at $t=\varepsilon$, the limit of $\dot{E}_{K L}$ from the left side is $m_{K L}$, while its limit from the right is $-m_{K L}$. In accordance with the criteria in (2.4), the cycle described by (3.1) and (3.2) involves loading along the straight line of length $\varepsilon$ which joins $E_{K L}^{0}$ and the point $E_{K L}^{*}=E_{K L}^{0}+\varepsilon m_{K L}$. At time $t=\varepsilon$, the direction of traversal is reversed, and elastic behavior ensues until the point $E_{K L}^{0}$ is reached again at time $t=2 \varepsilon$.

For the above cycle, with the help of (2.7) the inequality (2.10) can be written as

$$
H_{1}(\varepsilon)-H_{2}(\varepsilon)+K(\varepsilon) \leqslant 0,
$$

${ }^{5}$ It is worth emphasizing that (2.11) holds for all motions, and not just for homogeneous motions with homogeneous initial values of $\left(E_{M N}, E_{M N}, \kappa\right)$. For a discussion of this point, see [6, p. 40] or [10, p. 63]. 
where for convenience we have set

$$
\begin{gathered}
H_{1}(\varepsilon)=\int_{0}^{\varepsilon} h(t) t d t, \quad H_{2}(\varepsilon)=\int_{\varepsilon}^{2 \varepsilon} h(t)(2 \varepsilon-t) d t, \\
K(\varepsilon)=\int_{0}^{\varepsilon} k(t) t d t,
\end{gathered}
$$

with

$$
h(t)=L_{K L M N} m_{K L} m_{M N}, \quad k(t)=\lambda \sigma_{K L} \frac{\partial g}{\partial E_{M N}} m_{K L} m_{M N} .
$$

The functions $L_{K L M N}, \lambda, \sigma_{K L}, \partial g / \partial E_{M N}$ in (3.6) are understood to be parametrized by time, while $m_{K L}$ is independent of time.

Assuming sufficient smoothness, we apply Taylor's Theorem to the functions in (3.4) to obtain

$$
\begin{aligned}
H_{1}(0)-H_{2}(0)+K(0)+\left\{H_{1}^{\prime}(0)-H_{2}^{\prime}(0)+K^{\prime}(0)\right\} \varepsilon \\
+\left\{H_{1}^{\prime \prime}(0)-H_{2}^{\prime \prime}(0)+K^{\prime \prime}(0)\right\} \frac{\varepsilon^{2}}{2}+O\left(\varepsilon^{3}\right) \leqslant 0,
\end{aligned}
$$

where $O$ is the usual order symbol. The derivatives of the functions in (3.5) may be calculated with the use of the Fundamental Theorem of integral calculus and Leibniz's Formula, ${ }^{6}$ and are given by

$$
\begin{aligned}
& H_{1}^{\prime}(\varepsilon)=h(\varepsilon) \varepsilon, \quad H_{1}^{\prime \prime}(\varepsilon)=h(\varepsilon)+h^{\prime}(\varepsilon) \varepsilon, \\
& K^{\prime}(\varepsilon)=k(\varepsilon) \varepsilon, \quad K^{\prime \prime}(\varepsilon)=k(\varepsilon)+k^{\prime}(\varepsilon) \varepsilon, \\
& H_{2}^{\prime}(\varepsilon)=2 \int_{\varepsilon}^{2 \varepsilon} h(t) d t-h(\varepsilon) \varepsilon, \quad H_{2}^{\prime \prime}(\varepsilon)=4 h(2 \varepsilon)-3 h(\varepsilon)-h^{\prime}(\varepsilon) \varepsilon .
\end{aligned}
$$

Setting $\varepsilon=0$ in (3.5), (3.6) and (3.8), we find that

$$
\begin{aligned}
& H_{1}(0)=H_{2}(0)=K(0)=0, \quad H_{1}^{\prime}(0)=H_{2}^{\prime}(0)=K^{\prime}(0)=0, \\
& H_{1}^{\prime \prime}(0)=H_{2}^{\prime \prime}(0)=h(0)=\left.L_{K L M N}\right|_{t=0} m_{K L} m_{M N}, \\
& K^{\prime \prime}(0)=k(0)=\left.\left\{\lambda \sigma_{K L} \frac{\partial g}{\partial E_{M N}}\right\}\right|_{t=0} m_{K L} m_{M N} .
\end{aligned}
$$

Substitution of (3.9) into (3.7) leads to

$$
\left.\left\{\lambda \sigma_{K L} \frac{\partial g}{\partial E_{M N}}\right\}\right|_{t=0} m_{K L} m_{M N} \frac{\varepsilon^{2}}{2}+O\left(\varepsilon^{3}\right) \leqslant 0 .
$$

Dividing (3.10) by $\varepsilon^{2} / 2$ and taking the limit as $\varepsilon$ tends to zero, we obtain

$$
\left.\left\{\lambda \sigma_{K L} \frac{\partial g}{\partial E_{M N}}\right\}\right|_{t=0} m_{K L} m_{M N} \leqslant 0 .
$$

Hence, invoking (3.1) and $(2.6)_{2}$, we deduce that at any point on the yield surface

$$
\sigma_{K L} m_{K L} \leqslant 0
$$

${ }^{6}$ See, for example, Sec. 23 of Bartle [13]. 
for all $m_{K L}$ satisfying (3.1). Since $\sigma_{K L}$ is independent of $m_{K L}$, it follows readily ${ }^{7}$ from (3.12) that $\sigma_{K L}$ must satisfy (2.11).

4. Results for a special class of elastic-plastic materials. Prior to discussing a special set of constitutive equations, we first note that if the response function $\hat{S}_{K L}$ in (2.1) is invertible at fixed values of $E_{M N}$ and $\kappa$, then a yield function $f$ in stress space may be defined by [11]

$$
f\left(S_{K L}, E_{k L}, \kappa\right)=g\left(E_{M N}, E_{M N}, \kappa\right) .
$$

The equation $f=0$ then represents the five-dimensional yield surface in stress space, and $f<0$ corresponds to the elastic region in stress space. Also, at $g=f=0$, the normal to the yield surface in strain space is related to the normal to the yield in stress space by

$$
\frac{\partial g}{\partial E_{M N}}=L_{K L M N} \frac{\partial f}{\partial S_{K L}} .
$$

The latter equation may be solved for $\partial f / \partial S_{K L}$ by multiplying both sides by the partial derivatives of the inverse of $\hat{S}_{K L}$, taken with respect to $E_{M N}$.

Consider now a special stress response which is linear in the expression $E_{M N}-E_{M N}^{P_{N}}$ but with coefficients that depend on $E_{P_{Q}}$ and $\kappa$ :

$$
S_{K L}=L_{K L M N}\left\{E_{M N}-E_{M N}^{P_{N}}\right\},
$$

with

$$
L_{K L M N}=\hat{L}_{K L M N}\left(E_{P_{Q}}, \kappa\right)=L_{M N K L} .
$$

Il'iushin's development [3] is based on a constitutive equation of the type (4.3a) but without the dependency on $\kappa$. We may calculate the derivatives

$$
\dot{S}_{K L}=\dot{L}_{K L M N}\left\{E_{M N}-E_{M N}^{P_{N}}\right\}+L_{K L M N}\left\{\dot{E}_{M N}-\dot{E}_{M N}\right\}
$$

and

$$
\begin{gathered}
\frac{\partial \hat{S}_{K L}}{\partial E_{M N}}=\frac{\partial \hat{L}_{K L P Q}}{\partial E_{M N}^{P_{N}}}\left\{E_{P Q}-E \beta_{Q}\right\}-L_{K L M N}, \\
\frac{\partial \hat{S}_{K L}}{\partial \kappa}=\frac{\partial \hat{L}_{K L P Q}}{\partial \kappa}\left\{E_{P Q}-E P_{Q}\right\} .
\end{gathered}
$$

During unloading and neutral loading, as well as in an elastic state, (4.4) reduces to the form (2.7a).

4.1. Normality. Substitution of (4.5) into (2.8) gives

$$
\sigma_{K L}=-L_{K L M N} \rho_{M N}+\left(E_{P Q}-E \beta_{Q}\right)\left\{\frac{\partial \hat{L}_{K L P Q}}{\partial E_{M N}}+\frac{\partial \hat{L}_{K L P Q}}{\partial \kappa} \bigodot_{M N}\right\} \rho_{M N} .
$$

\footnotetext{
${ }^{7}$ See Sec. 5 of [6] for this detail.
} 
Multiplication of both sides of (4.6) by $\lambda \hat{g}$ leads with the aid of (2.4d) and (2.5) to the following expression which holds during loading:

$$
\begin{aligned}
\lambda \hat{g} \sigma_{K L} & =-L_{K L M N} \dot{E}_{M N}+\left(E_{P Q}-E P_{Q}\right)\left\{\frac{\partial \hat{L}_{K L P Q}}{\partial E_{M N}^{P}} \dot{E}_{M N}^{P_{N}}+\frac{\partial \hat{L}_{K L P Q}}{\partial \kappa} \dot{\kappa}\right\} \\
& =-L_{K L M N} \dot{E}_{M N}+\dot{L}_{K L M N}\left\{E_{M N}-E_{M N}^{p}\right\} .
\end{aligned}
$$

If (4.4) and (2.11) are used in (4.7) $)_{2}$, the resulting equation coincides with the normality condition originally given by Il'iushin [3]. ${ }^{8}$ It should be noted that while Il'iushin [3] considers a constitutive equation of the form (4.3a)-but without the dependency on $\kappa$-his discussion is confined to small deformations. In particular, Il'iushin [3] identifies the term $E_{K L}-E_{K L}^{R_{L}}$ with elastic strain. No assumption regarding smallness of deformation has been made in the present section, and the results hold in the presence of finite deformation.

In the remainder of this section, we will suppose that $L_{K L M N}$ are constants. Then by (2.11) and (4.6)

$$
L_{K L M N} \rho_{M N}=\gamma^{*} \frac{\partial g}{\partial E_{K L}}, \quad \gamma^{*}>0,
$$

where the inequality follows from the invertibility ${ }^{9}$ of $L_{K L M N}$ and the fact that not all components of $\rho_{M N}$ vanish. Hence, by (4.2) and (4.3b)

$$
\rho_{K L}=\gamma^{*} \frac{\partial f}{\partial S_{K L}}
$$

and therefore, in view of $(2.4 \mathrm{~d})$,

$$
\dot{E}_{K L}^{p}=\lambda \gamma^{*} \hat{g} \frac{\partial f}{\partial S_{K L}}
$$

during loading ${ }^{10}$. Thus, the plastic strain rate is directed along the normal to the yield surface in stress space. With $\rho_{K L}$ satisfying (4.8b), (2.6) can be solved for the product $\lambda \gamma^{*}$.

We observe that if both sides of (4.8a) $)_{1}$ are contracted with $\lambda \hat{g} \dot{E}_{K L}$ and use is made of (2.3), (2.4d), $(2.6)_{2}$ and (4.8a) $)_{2}$, it may be deduced that during loading

$$
L_{K L M N} \dot{E}_{K L} \dot{E}_{M N}>0 \text {. }
$$

Noting from (4.3a) that $\dot{S}_{K L}=L_{K L M N}\left\{\dot{E}_{M N}-\dot{E}_{M N}\right\}$, we can immediately establish an inequality which has the same form as (2.13) except for the equality sign, and invoking (4.3b) we may also obtain the complementary inequality

$$
\dot{S}_{K L} \dot{E}_{K L}^{R_{L}}>-L_{K L M N} \dot{E}_{K L}^{R} \dot{E}_{M N}^{p} .
$$

Results of the type (2.13), (4.10) and (4.11) (with equality signs added) were derived by Naghdi \& Trapp [10] for the case of constant $L_{K L M N}$. For a discussion of the significance of these inequalities, the reader is referred to $[10,12]$.

\footnotetext{
${ }^{8}$ See Eqs. (8) and (9) of [3].

${ }^{9}$ I.e., as a linear transformation on the six-dimensional space of symmetric second order tensors.

${ }^{10}$ The normality conditions (4.8) and (4.9) also hold under less restrictive conditions than the constancy of the fourth order tensor in (4.3a). See $[6,10,12]$.
} 
4.2. Convexity. For the case of constant $L_{K L M N}$, both the yield surface in strain space and the yield surface in stress space are convex [10]. It is instructive to establish this result by a variation of the proof given in Sec. 3 .

First observe that when $L_{K L M N}$ are constants, the inequality (2.10) may, with the aid of (4.4), be written as [10]

$$
\begin{aligned}
\int_{t_{1}}^{t_{2}} \frac{d}{d t}\left[\frac{1}{2} L_{K L M N}\right. & \left.\left\{E_{M N}-E_{M N}^{0}\right\}\left\{E_{K L}-E_{K L}^{0}\right\}\right] d t \\
& -\int_{t_{1}}^{t_{2}} L_{K L M N} \dot{E}_{M N}^{p_{N}}\left\{E_{K L}-E_{K L}^{0}\right\} d t \leqslant 0 .
\end{aligned}
$$

The first integral in (4.12) vanishes, its value at both the beginning and end of the cycle being zero. Imagine any strain trajectory beginning at $E_{K L}^{0}$, and intersecting the yield surface for the first time, which we may take to be $t=0$, at the point $E_{K L}^{y}$. Let the line segment joining $E_{K L}^{0}$ to $E_{K L}^{k}$ be expressed as

$$
E_{K L}^{k}-E_{K L}^{0}=\xi n_{K L} \quad\left(n_{K L} n_{K L}=1, \xi \geqslant 0\right) .
$$

Let the cycle be continued along the trajectory

$$
E_{K L}(t)=E_{K L}^{\ddot{k}}+t m_{K L}, \quad 0 \leqslant t \leqslant \varepsilon,
$$

with $m_{K L}$ and $\varepsilon$ satisfying the same conditions as in Sec. 3. Finally, let the cycle be closed by any strain trajectory that joins the point $E_{K L}^{*}=E_{K L}^{v}+\varepsilon m_{K L}$ to $E_{K L}^{0}$ and does not involve loading. A cycle of the type just described is illustrated in Fig. 1.

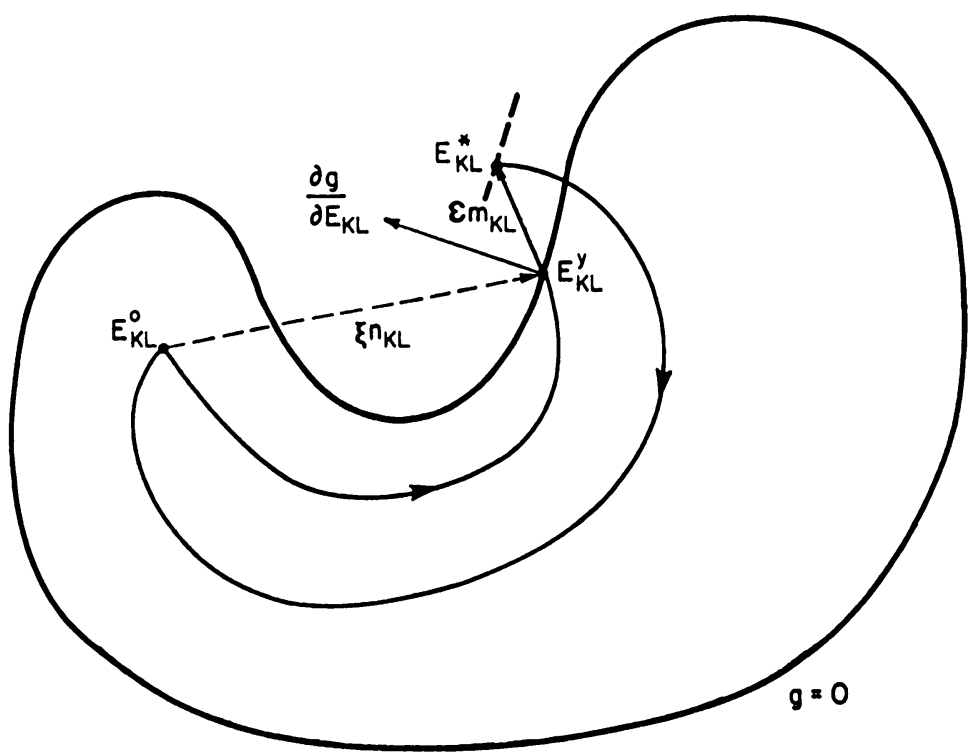

FIG.1. A sketch of a hypothetical yield surface in strain space together with a strain cycle of the type employed in the convexity proof. The cycle begins at $E_{K L}^{0}$ and first intersects the yield surface at $E_{K L}^{v}$. It continues along a straight line segment of length $\varepsilon$ pushing the yield surface outwards to $E_{K L}^{*}$. The cycle then closes by any path that joins $E_{K L}^{*}$ to $E_{K L}^{0}$ and lies in the new elastic region. In the cycle, loading occurs only along the segment joining $E_{K L}^{v}$ to $E_{K L}^{*}$. 
With the aid of (2.4d), the inequality (4.12) reduces to

$$
-\int_{0}^{\varepsilon} \lambda L_{K L P Q} \rho_{P Q} \frac{\partial g}{\partial E_{M N}} \dot{E}_{M N}\left\{E_{K L}-E_{K L}^{0}\right\} d t \leqslant 0 .
$$

Hence, using (4.13) and (4.14), we may write (4.15) in the form

$$
K(\varepsilon)+\bar{K}(\varepsilon) \leqslant 0,
$$

where $K(\varepsilon)$ is defined in $(3.5)_{3}$, with $k(t)$ in $(3.6)_{2}$ being now given by ${ }^{\prime \prime}$

$$
k(t)=-\lambda L_{K L P Q} \rho_{P Q} \frac{\partial g}{\partial E_{M N}} m_{K L} m_{M N} .
$$

The function $\bar{K}(\varepsilon)$ in (4.16) is defined by

$$
\bar{K}(\varepsilon)=\xi \int_{0}^{\varepsilon} \bar{k}(t) d t
$$

with

$$
\bar{k}(t)=-\lambda L_{K L P Q} \rho_{P Q} \frac{\partial g}{\partial E_{M N}} n_{K L} m_{M N} .
$$

The first and second derivatives of $K(\varepsilon)$ have already been calculated in $(3.8)_{3,4}$. Similarly,

$$
\bar{K}^{\prime}(\varepsilon)=\xi \bar{k}(\varepsilon), \quad \bar{K}^{\prime \prime}(\varepsilon)=\xi \bar{k}^{\prime}(\varepsilon) .
$$

The values at $\varepsilon=0$ of $K(\varepsilon)$ and its first and second derivatives are given in (3.9), while from (4.18) and (4.20) we obtain

$$
\bar{K}(0)=0, \quad \bar{K}^{\prime}(0)=\xi \bar{k}(0), \quad \bar{K}^{\prime \prime}(0)=\xi \bar{k}^{\prime}(0) .
$$

A Taylor expansion of (4.16) about $\varepsilon=0$ then implies that

$$
\xi \bar{k}(0) \varepsilon+\left\{k(0)+\xi \bar{k}^{\prime}(0)\right\} \frac{\varepsilon^{2}}{2}+O\left(\varepsilon^{3}\right) \leqslant 0,
$$

and hence that

$$
\xi \bar{k}(0)+\left\{k(0)+\xi \bar{k}^{\prime}(0)\right\} \frac{\varepsilon}{2}+O\left(\varepsilon^{2}\right) \leqslant 0 .
$$

If $\xi$ is chosen to zero in (4.13) then from (4.23) we obtain the result $k(0) \leqslant 0$, which was established for general constitutive equations in Sec. 3. Consequently, as would be expected, the normality condition (4.8a) follows from the present proof when $\xi=0$.

Taking $\xi>0$ and letting $\varepsilon$ approach zero in (4.23), we deduce that

$$
\bar{k}(0) \leqslant 0 \text {. }
$$

It then follows with the help of (4.19), $(2.6)_{2},(3.1)$ and (4.8a) that on the yield surface in strain space

$$
\frac{\partial g}{\partial E_{K L}} n_{K L} \geqslant 0
$$

\footnotetext{
"On purpose, we do not invoke the normality condition (4.8a), because it is interesting to see how it emerges as part of the present proof.
} 
or, recalling (4.13), that

$$
\frac{\partial g}{\partial E_{K L}}\left\{E_{K L}^{y}-E_{K L}^{0}\right\} \geqslant 0 .
$$

Since $E_{K L}^{0}$ may be chosen anywhere in the region $g<0$, and $E_{K L}^{\nu}$ can be any point on the yield surface $g=0$, the inequality (4.25), or equivalently (4.26), ensures convexity of the yield surface in strain space. ${ }^{12}$

The convexity of the yield surface in stress space follows from (4.1), (4.2), (4.3a) and (4.26). Thus, for the same value of plastic strain, let $S_{M N}^{0}$ be the stress corresponding to $E_{K L}^{0}$. Then $S_{M N}^{0}$ lies in the region $f<0$ and $S_{M N}^{y}$ is on the yield surface $f=0[11,7]$. Conversely, if $S_{M N}^{0}$ is any point in the region $f<0$, the corresponding strain $E_{K L}^{0}$ is in the region $g<0$, and if $S_{M N}^{v}$ is any point on $f=0$, the corresponding strain $E_{K L}^{v}$ is on $g=0$. The inequality (4.26) therefore leads to the conditon that

$$
\frac{\partial f}{\partial S_{M N}}\left\{S_{M N}^{y}-S_{M N}^{0}\right\} \geqslant 0
$$

for all $S_{M N}^{y}$ on $f=0$ and $S_{M N}^{0}$ in $f<0$, which implies convexity.

\section{REFERENCES}

[1] D. C. Drucker, A more fundamental approach to plastic stress-strain relations, Proc. First U.S. National Cong. of Appl. Mech. (Chicago, 1951), 487-491, ASME, 1952

[2] P. M. Naghdi, Stress-strain relations in plasticity and thermoplasticity, Proc. Second Symp. on Naval Structural Mech. (Brown University, 1960), 121-169, Pergamon Press, 1960

[3] A. A. Il'iushin, On the postulate of plasticity, J. Appl. Math. Mech. (Translation of Prikladnaya Mathematika i Mekhanika) 25, 1961, 746-752

[4] A. E. Green \& P. M. Naghdi, A general theory of an elastic-plastic continuum, Arch. Ratl. Mech. Anal. 18. $1965,251-281$

[5] __ A thermodynamic development of elastic-plastic continua, Proc. IUTAM Symp. on Irreversible Aspects of Continuum Mech. and Transfer of Physical Characteristics in Moving Fluids (ed. H. Parkus \& L. I. Sedov) 117-131, Springer-Verlag, 1966

[6] P. M. Naghdi \& J. A. Trapp, Restrictions on constitutive equations of finitely deformed elastic-plastic materials, Quar. J. Mech. Appl. Math. 28, 1975, 25-46

[7] J. Casey \& P. M. Naghdi, On the characterization of strain-hardening in plasticity, J. Appl. Mech. 48, 1981, 285-296

[8] _ A remark on the definition of hardening, softening and perfectly plastic behavior. Acta Mech. 48, $1983,91-94$

[9] __ Strain-hardening response of elastic-plastic materials, Presented at International Conf. on Constitutive Laws for Engineering Materials: Theory and Application (Tucson, Arizona, 10-14 January, 1983); To appear in Mechanics of Engineering Materials, Wiley

[10] P. M. Naghdi \& J. A. Trapp, On the nature of normality of plastic strain rate and convexity of yield surfaces in plasticity, J. Appl. Mech. 42, 1975, 61-66

[11] __ The significance of formulating plasticity theory with reference to loading surfaces in strain space, Int. J. Engng. Sci. 13, 1975, 785-797

[12] P. M. Naghdi, Some constitutive restrictions in plasticity, In Constitutive equations in viscoplasticity: computational and engineering aspects, AMD, 20, 79-93, ASME., 1976

[13] R. G. Bartle, The elements of real analysis, Wiley, 1964

\footnotetext{
${ }^{12}$ For additional remarks on convexity, see [2, p. 135].
} 